\title{
ASSOCIAÇÃO ENTRE A AUTOPERCEPÇÃO DO ENVELHECIMENTO E A AUTOPERCEPÇÃO DA SAÚDE?
}

\author{
Patrícia Galdino de Andrade Wollmann² \\ Stéfane Araújo Coelho ${ }^{3}$ \\ Laura Garcia Boaventura ${ }^{4}$ \\ Beatriz Guimarães Murici5 \\ Geórgia Danila Fernandes D'Oliveira ${ }^{6}$ \\ Gislane Ferreira de Melo?
}

resumo

A autopercepção da saúde é um determinante multidimensional e subjetivo do indivíduo para análise de sua própria saúde, considerado um fator para evidências de declínio funcional e de mortalidade. 0 objetivo deste estudo foi verificar a associação da autopercepção do envelhecimento e autopercepção da saúde. Trata-se de um estudo com delineamento transversal, descritivo e de associação. A amostra foi constituída de 224 indivíduos com idade igual ou superior a 60 anos, de ambos os sexos, sendo 64,4\% indivíduos femininos e 35,5\% masculinos, todos residentes no Distrito Federal. A coleta

1 Pesquisa de financiamento próprio.

2 Graduada e Licenciada em Educação Física. Graduada em Enfermagem. Doutoranda em Gerontologia pela Universidade Católica de Brasília. Professora e Coordenadora da Faculdade LS. E-mail: patricia.wollmanndf@gmail.com.

3 Graduada em Enfermagem pela Faculdade LS. E-mail: sac.stefanecoelho@gmail.com,

4 Graduada em Enfermagem pela Faculdade LS. E-mail: lauragarcia.b.g@hotmail.com.

5 Graduada em Enfermagem pela Faculdade LS. E-mail: beatriz.gmurici@gmail.com.

6 Graduada em Fisioterapia. Doutora em Educação Física. Coordenadora do Curso de Fisioterapia do Centro Universitário Projeção. E-mail: georgiadanila@gmail.com.

7 Graduada em Educação Física. Doutora em Educação Física. Coordenadora do Programa Stricto Sensu em Educação da Universidade Católica de Brasilia. E-mail: gislane.melo@gmail.com. 
de dados foi realizada por meio de questionário sociodemográfico e pelo Questionário de Autopercepção do Envelhecimento (QAPE), versão brasileira. Foram realizadas análises descritivas, comparativas de grupo e correlações. Verificou-se que não ocorreu diferença estatística significativa entre a autopercepção do envelhecimento e a autopercepção da saúde, contudo, os dados demonstram que quanto melhor a autopercepção da saúde, melhor a autopercepção do envelhecimento.

palavras-chave

Autopercepção. Saúde. Envelhecimento. Idoso.

\section{Introdução}

O envelhecimento da população é um fenômeno de amplitude mundial. O número de idosos, antes mais alto em países desenvolvidos, vem aumentando também nos países em desenvolvimento e de modo mais acentuado. Atualmente, cerca de dois terços das pessoas mais velhas do mundo vivem em países em desenvolvimento (UNITED NATIONS, 2009; MIRANDA; MENDES; SILVA, 2016).

As alterações características do processo de envelhecer afetam naturalmente o processo do padrão de saúde/doença do indivíduo. O avanço da medicina e da tecnologia tem originado um aumento da expectativa de vida, acompanhado da diminuição das taxas de natalidade e mortalidade e, consequentemente, gerado um crescimento significativo do envelhecimento da população mundial (FECHINE; TROMPIERI, 2012; MOREIRA et al., 2013; JEREZ-ROIG et al., 2016).

O processo de envelhecimento é acompanhado de uma variedade de perdas e ganhos, consequentemente, aspectos como a crise de identidade, mudança de papéis sociais, aposentadoria e perdas morfofisiológicas tornaram-se um dos maiores desafios de saúde pública para essa população (NUNES; BARRETO; GONÇALVES, 2012). Essas perdas podem promover a insatisfação com o processo e a velhice, bem como uma diminuição da autoestima geral, levando a morbidades e doenças psicológicas (ROCHA; VIEBIG; LATTERZA, 2012).

Durante o processo de envelhecimento, constata-se uma maior predisposição do indivíduo às doenças crônicas, entretanto, as doenças e limitações não 
são consequências inevitáveis do envelhecimento (RIBEIRO; ALVES; MEIRA, 2009). Assim, nessa etapa, ocorre uma diminuição da capacidade funcional que muitas vezes não gera incapacidade, porém traz algumas limitações à pessoa (FIEDLER; PERES, 2008).

Com o passar da idade, as limitações e os problemas de saúde mostram-se mais evidentes, revelando uma frequência de percepção de saúde negativa, o que pode interferir no bem-estar e na qualidade de vida dos idosos (JEREZ-ROIG et al., 2016). Contudo, a maneira como as pessoas percebem seu próprio envelhecimento e estado de saúde pode ser um indicador importante para sua adaptação e bem-estar, consequentemente, o indivíduo pode apresentar percepções positivas, o que pode ser fator determinante para um envelhecimento bem-sucedido (FERREIRA et al., 2012).

A autopercepção da saúde considera aspectos de saúde física, cognitiva e emocional (OFSTEDAL et al., 2002; CONFORTIN et al., 2015). No idoso, o julgamento sobre sua saúde se estabelece dentro de uma conjuntura que abrange as alterações físicas e mentais próprias ao processo de envelhecimento, integrada às questões culturais e ambientais nas quais o idoso está inserido (FREITAS et al., 2010).

Dessa maneira, a avaliação de percepções quanto ao envelhecimento proporciona observar não só o diagnóstico precoce de problemas de saúde, mas também antecipar possíveis fatores que possam influenciar negativamente a qualidade de vida do indivíduo na velhice e na sua saúde (ROCHA; GOMES; SCHWANKE, 2012). Portanto, o objetivo desse estudo foi verificar a associação da autopercepção do envelhecimento e autopercepção da saúde.

\section{Materiais e métodos}

Trata-se de uma pesquisa transversal, quantitativa e descritiva. O estudo foi realizado no período de fevereiro a maio de 2015, com idosos participantes do Centro de Convivência da Universidade Católica de Brasília, Distrito Federal, espaço onde os idosos realizam atividades recreativas, de lazer e culturais. Com o objetivo de respeitar integralmente os dispositivos expostos junto à Resolução n.o 466/2012 do Conselho Nacional de Saúde (CNS), o presente projeto foi submetido e aprovado pelo Comitê de Ética e Pesquisa, sob o Parecer n.o 692.959/2014.

A amostra foi aleatória e participaram da pesquisa 224 idosos (80 homens e 144 mulheres) com média de idade igual a 69,73 + 7,62 anos. Foram incluídas no estudo pessoas com idade igual ou superior a 60 anos, residentes no 
Distrito Federal e de acordo em participar da pesquisa, assinando o Termo de Consentimento Livre e Esclarecido (TCLE), e em responder aos dois questionários aplicados.

O critério de exclusão consistia em idosos que não tinham condições de responder aos questionários. Foi utilizada para técnica de coleta de dados a abordagem aos idosos explicando o objetivo da pesquisa e a forma como seria executada. A entrevista foi realizada individualmente. Logo após a explicação, os idosos responderam à entrevista por meio do questionário sociodemográfico, desenvolvido pelos próprios pesquisadores e composto por 18 questões, classificando as informações em: a) Identificação do indivíduo avaliado (local da residência, naturalidade, atividade profissional, idade, sexo, estado civil, religião); b) Identificação do perfil socioeconômico (quantidade de filhos, escolaridade, moradia, renda); e c) Identificação do estado de saúde (autoavaliação do estado de saúde, se passou por internação hospitalar recente, qual tipo de serviço de saúde utiliza, patologias conhecidas e se utiliza medicamento regularmente e quantos).

O segundo instrumento utilizado foi o Aging Perceptions Questionnaire-APQ, um instrumento importante para a identificação do envelhecimento bem-sucedido (BARKER et al., 2007). Esse instrumento foi adaptado por Rocha, Gomes e Schwanke (2012) para a língua portuguesa do Brasil, ficando conhecido no país como Questionário de Autopercepção do Envelhecimento (QAPE). O QAPE foi apresentado aos idosos, sujeitos da amostra, com o objetivo de mensurar a autopercepção do envelhecimento, visto ser este construto importante para a identificação do envelhecimento bem-sucedido (BARKER et al., 2007).

O QAPE é dividido em dois questionários, onde o primeiro infere as opiniões sobre o envelhecimento, sendo constituído de 32 itens que tem como intuito verificar quantitativamente a percepção de envelhecimento por meio de quatro dimensões: (1) Cronologia (crônica e cíclica): consciência do envelhecimento e a variação na experiência deste processo através do tempo. (2) Consequências (positivas e negativas): valores e crenças sobre os impactos positivos e negativos do envelhecimento na vida dos indivíduos. (3) Controle (positivo e negativo): crenças sobre o poder dos indivíduos sobre os aspectos tanto positivos quanto negativos do envelhecimento. (4) Representações Emocionais: respostas emocionais ao envelhecimento (ROCHA; GOMES; SCHWANKE, 2012).

Para a caracterização da amostra, foi utilizada a estatística descritiva, com demonstração de valores de média e desvio-padrão, por meio do software Statistical Package for Social Science - SPSS 22.0 for Windows. As análises inferenciais foram realizadas com o intuito de avaliar o grau de significância de 
algumas das diferenças encontradas nos cruzamentos dos dados. Admitiu-se significância estatística entre as variáveis cujo p-valor fosse menor que 0,05 $(\mathrm{p}<0,05)$. Dentre os testes utilizados, o qui-quadrado (análise estatística não paramétrica) foi realizado para verificar diferenças nas percepções de envelhecimento e saúde entre homens e mulheres.

\section{Resultados}

Participaram da amostra 224 idosos, com média de idade para os homens de 70,65 $\pm 8,24$ anos e, para as mulheres, de 69,22 $\pm 7,24$ anos, sendo a idade mínima igual a 60 anos e a máxima 94 anos para ambos. Foram analisados $144(64,4 \%)$ mulheres e $80(35,6 \%)$ homens. As características sociodemográficas dos idosos estão apresentadas na Tabela 1 . 
Tabela 1 - Dados sociodemográficos de 224 idosos do Distrito Federal, 2015.

\begin{tabular}{lllll}
\hline \multirow{2}{*}{ Variáveis } & \multicolumn{2}{c}{ Homens } & \multicolumn{2}{c}{ Mulheres } \\
\cline { 2 - 5 } & $\mathrm{n}$ & $\%$ & $\mathrm{n}$ & $\%$ \\
\hline Faixa etária & 43 & 53,8 & 84 & 58,3 \\
$60-69$ & 25 & 31,2 & 44 & 30,6 \\
$70-79$ & 12 & 15,0 & 10 & 11,1 \\
$80+$ & & &
\end{tabular}

\section{Estado civil}

Casado

62

77,5

60

41,7

Solteiro

3,8

11

7,6

Divorciado

3

6,3

22

15,3

Viúvo

11,3

32,6

Outro

1,3

47

2,1

\section{Escolaridade}

Não Alfabetizado

Alfabetizado

4

5

6,3

Fundamental

20

25

16,7

Médio

25

31,3

28,5

Superior

\section{Religião}

Católica

Evangélica

58

72,5

55,6

Espírita

Outra

\section{Faz uso de medicamentos}

Sim

Não

\section{Número de doenças}

\begin{tabular}{lllll}
0 & 13 & 16,3 & 1 & 0,7 \\
1 & 26 & 32,5 & 29 & 20,1 \\
2 & 13 & 16,3 & 35 & 24,3 \\
3 & 15 & 18,8 & 33 & 22,9 \\
4 & 2 & 2,5 & 16 & 11,1 \\
5 & 1 & 1,3 & 11 & 7,6 \\
6 & 4 & 5,0 & 5 & 3,5 \\
7 & 0 & 0 & 3 & 2,1 \\
8 & 0 & 0 & 2 & 1,4 \\
Total de participantes & 80 & & 144 & \\
\hline
\end{tabular}

Fonte: Dados da pesquisa (2015). 
Para verificar diferenças nas percepções entre homens e mulheres, foram realizadas análises como o teste t para amostras independentes. Dessa forma, a variável sexo foi analisada com as dimensões do Questionário de Autopercepção do Envelhecimento, para essas análises nenhum resultado foi significativo (Tabela 2). Na análise da experiência com mudanças relacionadas à saúde durante o envelhecimento, com referência de 0 a 100, observou-se uma percepção acima de 70 para homens e para mulheres, como mostrado na Tabela 2.

Tabela 2 - Teste t para amostras independentes da autopercepção do envelhecimento, Distrito Federal, 2015.

\begin{tabular}{llll}
\hline Dimensões & Homens & Mulheres & $p$ \\
\hline Crônica & $3,43(0,59)$ & $3,45(0,72)$ & 0,815 \\
Cíclica & $2,54(0,61)$ & $2,49(0,83)$ & 0,659 \\
Consequências positivas & $4,05(0,82)$ & $4,18(0,85)$ & 0,264 \\
Consequências negativas & $3,12(0,74)$ & $3,03(0,81)$ & 0,446 \\
Controle positivo & $3,91(0,63)$ & $3,90(0,75)$ & 0,978 \\
Controle negativo & $2,96(0,77)$ & $3,00(0,89)$ & 0,750 \\
Representações & $2,22(0,77)$ & $2,23(0,81)$ & 0,921 \\
Percepção da saúde & $70,60(32,92)$ & $75,08(26,14)$ & 0,265 \\
\hline
\end{tabular}

Fonte: Dados da pesquisa (2015).

Sobre a percepção da saúde, $12 \%(\mathrm{n}=27)$ referiu à saúde como excelente, $52,4 \%(n=118)$ como boa, $32 \%(n=72)$ como razoável e $2,7 \%(n=6)$ como ruim. Embora a maioria dos idosos mencione a saúde como boa, uma grande maioria refere ser portadora de doenças crônicas ou fazer uso de medicação sistematicamente.

Quanto às doenças crônicas mais frequentes, 59,8\% da amostra relatou ter hipertensão arterial sistêmica, 33,9\% colesterol alto, 20,1\% triglicérides e reumatismo, 18,3\% são diabéticos, entre outras doenças. Com relação à utilização de medicamentos, os indivíduos que utilizam medicação regularmente correspondem a $82,7 \%$ da amostra, com média de 2,97 $\pm 2,73$ medicamentos. Cabe ressaltar que, destes, 51,5\% utilizam de 1 a 3 medicamentos, 22,3\% utilizam de 4 a 6 medicamentos e 9,7\% utilizam 7 ou mais medicamentos por dia.

Nas informações referentes às condições clínicas, foram realizadas análises inferenciais, como o teste qui-quadrado, para analisar a frequência de percepção baixa, moderada e alta relacionada à percepção da saúde, de acordo com o percentil encontrado na tabulação cruzada entre idosos com e sem doenças (Tabela 3). 
Tabela 3 - Percentis da percepção da saúde relacionadas à presença ou ausência de doenças, Distrito Federal, 2015.

\begin{tabular}{llll}
\hline & \multicolumn{3}{l}{ Percepção da saúde } \\
& Baixa & Moderada & Alta \\
\hline Sem doenças & $37 \%$ & $22,2 \%$ & $40,7 \%$ \\
Com doenças & $18,8 \%$ & $28,9 \%$ & $52,3 \%$ \\
\hline \multicolumn{5}{c}{$p=0,092$} \\
\hline
\end{tabular}

Fonte: Dados da pesquisa (2015).

Para verificar diferenças nas percepções entre homens e mulheres, foram realizadas análises como o qui-quadrado. Desta forma, a variável sexo foi analisada com a percepção do processo de envelhecimento, para essas análises, nenhum resultado foi significativo, como demonstra a Tabela 4.

Tabela 4 - Relação da percepção do processo do envelhecimento com o sexo, Distrito Federal, 2015.

\begin{tabular}{lll}
\hline Percepção do processo do envelhecimento & Homens & Mulheres \\
\hline Excelente & $7,5 \%$ & $20,8 \%$ \\
Boa & $58,8 \%$ & $43,1 \%$ \\
Razoável & $27,5 \%$ & $28,5 \%$ \\
Ruim & $5,0 \%$ & $5,6 \%$ \\
Péssima & $0,0 \%$ & $0,0 \%$ \\
\hline
\end{tabular}

Fonte: Dados da pesquisa (2015).

Foi comparada a percepção do processo do envelhecimento com as classificações da percepção de saúde, e verificou-se que não ocorreu diferença estatística significativa, mostrada na Tabela 5. 
Tabela 5 - Relação da percepção do processo de envelhecimento e percepção da saúde, Distrito Federal, 2015.

\begin{tabular}{llll}
\hline \multirow{2}{*}{ Percepção do processo do envelhecimento } & \multicolumn{3}{l}{ Percepção da saúde } \\
& Baixa & Moderada & Alta \\
\hline Excelente & $10,6 \%$ & $11,1 \%$ & $21,1 \%$ \\
Boa & $55,3 \%$ & $44,4 \%$ & $48,2 \%$ \\
Razoável & $23,4 \%$ & $33,3 \%$ & $27,2 \%$ \\
Ruim & $6,4 \%$ & $9,5 \%$ & $2,6 \%$ \\
Péssima & $2,1 \%$ & $1,6 \%$ & $0,0 \%$ \\
\hline \multicolumn{5}{l}{} \\
\hline
\end{tabular}

Fonte: Dados da pesquisa (2015).

\section{Discussão}

A autopercepção da saúde influencia na autoavaliação do indivíduo a respeito de seu estado de saúde. No idoso, a autopercepção da saúde se estabelece dentro de um contexto que envolve as alterações biopsicossocioculturais próprias do processo de envelhecimento no qual ele está inserido (FREITAS et al., 2010).

O inquérito de Autopercepção de Saúde consiste em perguntar ao idoso sobre como ele percebe a sua própria saúde, o que esboça como o indivíduo percebe sua qualidade de vida. O presente estudo avaliou a percepção como "baixa", "moderada" ou "alta" e comparou-as entre os grupos da amostra com doenças e sem doenças. A percepção de saúde foi considerada alta por ambos os grupos, "idosos sem doenças", com 40,7\%, e "idosos com doenças", com 52,3\%. É interessante identificar que a percepção baixa de saúde em indivíduos sem doenças foi superior à encontrada nos indivíduos com doenças (37\% e 18,8\%, respectivamente), o que corrobora com a ideia de Borges et al. (2014) de que a percepção de saúde negativa não está atrelada a ser portador doenças, mas possuir incapacidades em decorrência delas. Independente de possuir ou não doenças, houve uma autopercepção da saúde considerada "alta" por parte dos entrevistados tanto sem doenças (40,7\%) quando com doenças (52,3\%).

A determinação da qualidade de vida, mesmo na presença de agravos e doenças, tem influência da autopercepção da saúde, que consiste em sentir-se bem ainda que com comorbidades. Dessa maneira, as doenças crônicas de longa 
permanência, desde que controladas, muitas vezes por fármacos, podem não comprometer a qualidade de vida em idosos (RABELO et al., 2010).

No presente estudo, constatou-se que quase 50\% dos indivíduos apresenta acima de três doenças. Em consonância com outros estudos que apresentam resultados semelhantes, como 52\% (CONFORTIN et al., 2015), 87,4\% (SILVA; PINTO JUNIOR; VILELA, 2014) e 56,7\% (CONFORTIN et al., 2017). As doenças mais prevalentes no estudo são hipertensão arterial, hiperlipidemias, reumatismo e diabetes mellitus. Dentre os entrevistados, 82,7\% afirma fazer uso de medicações regularmente. A presença de doenças crônicas não transmissíveis nos idosos muitas vezes requer a contínua utilização de vários fármacos (polifarmácia) para manutenção da saúde e casos de doenças.

O uso de medicamentos tem média de 2,97 $\pm 2,73$ e o uso de mais de três medicações é de 32,0\%, enquanto que nos achados do estudo de Silveira, Dalastra e Pagotto (2014) foi encontrado que a prevalência do uso de medicações em idosos da comunidade em Goiânia é de 3,7 $\pm 2,4$, sendo que o estudo considerou somente a polifarmácia, encontrada em 28,0\% da amostra. Apesar da terapêutica por meio do uso de medicamentos para controle de doenças crônicas, a mudança do estilo de vida como forma de tratamento não farmacológico é essencial para a melhoria da qualidade de vida desses idosos (SILVEIRA; DALASTRA; PAGOTTO, 2014). O estudo realizado por Cardoso et al. (2012) demonstra que idosos acometidos por doenças crônicas degenerativas e que têm um estado funcional diminuído possuem uma autopercepção da saúde consideravelmente baixa, mostrando que a autopercepção negativa está relacionada à falta de autonomia e dependência ocasionada pelas doenças.

Silva e Menezes (2007) entendem que um dos fatores para uma boa e adequada análise sobre a relação entre a autopercepção da saúde e a mortalidade nos idosos é o acesso da população às informações sobre saúde, que é comprometida nos países em desenvolvimento em comparação aos países desenvolvidos. Foram encontrados os seguintes achados no presente estudo que contribuem para essa informação: o maior quantitativo de homens tinha nível de escolaridade até o ensino fundamental $(31,3 \%)$ e de mulheres, o maior foi o de nível médio $(36,6)$, apresentando ainda um grupo significativo de homens apenas alfabetizados (25\%) e mulheres que possuem até o ensino fundamental $(28,5 \%)$.

Um estudo realizado por Guerra e Caldas (2010) constatou a importância das escolhas ao longo da vida, as possibilidades internas e o engajamento social como fatores que influenciam a autopercepção do envelhecimento. Os autores dizem que o interesse pelo relato dos idosos colabora para uma compreensão real do idoso, das mudanças percebidas por ele no seu ambiente social e no seu 
próprio corpo, a contar as alterações físicas e mentais, apesar da descrição das perdas e limitações advindas do processo do envelhecimento. Infere-se que há um significante impacto no envelhecimento ativo e em uma boa percepção da velhice quando se leva em consideração a opinião do idoso sobre seu próprio entendimento acerca desse processo.

A análise da autopercepção do idoso quanto ao envelhecimento é um fator determinante para a qualidade de vida e definição do estado de saúde do indivíduo. Quando analisadas as dimensões do QAPE, a maior incidência encontrada neste estudo foi nas escalas de Consequências Positivas e Controle Positivo para homens e mulheres. Os resultados obtidos parecem ser consonantes com aquilo que autores como Baker et al. (2007) e Rocha, Gomes e Schwanke (2012) observaram em suas pesquisas, no sentido em que o envelhecimento apresenta aspectos mais positivos do que negativos, sugerindo, assim, que o envelhecimento para essa população apresenta aspectos que podem agregar sabedoria e crescimento.

Em desencontro com o descrito na literatura, como, por exemplo, nos estudos de Borges et al. (2014) e de Silva e Menezes (2007), o presente estudo detectou uma autopercepção de envelhecimento superior das mulheres $(75,08$ - 26,14) em relação à autopercepção dos homens $(70,60$ - 32,92), porém não houve significância estatística $(\mathrm{p}=0,265)$. A presente pesquisa mostra uma tendência dos estudos brasileiros, que é uma maior prevalência de mulheres participantes da pesquisa. Estudos ainda apontam para a feminilização da velhice que, segundo Silva, Pinto Junior e Vilela (2014) pode ser atribuída à maior busca por serviços de saúde e interesse na própria saúde pelas mulheres.

Quando analisada a autopercepção da saúde, Barker et al. (2007) consideram que quanto melhor a percepção de saúde de um idoso, melhor sua autopercepção de envelhecimento, sendo encontrados os mesmos resultados nesta pesquisa. Em sua maioria, idosos que consideram a autopercepção do envelhecimento excelente consideram a autopercepção da saúde como "alta"; os que a descrevem como "boa" relatam uma "baixa" percepção de saúde e os que descrevem como "razoável" percebem sua saúde como "razoável". Esses achados corroboram com outros autores que descrevem que uma percepção de saúde negativa e uma autopercepção de envelhecimento estão relacionadas a múltiplos fatores, não só à presença ou não de doenças, visto que 195 idosos (87,05\%) possuem ao menos uma doença/comorbidade.

Medeiros et al. (2016) relatam ainda uma problemática presente nos estudos brasileiros e internacionais, revelando pluralidade e discordâncias relacionadas à análise das respostas acerca da autopercepção da saúde, e uma não padronização das metodologias, mesmo utilizando o mesmo instrumento, 
visto que o estudo dos autores supracitados mostra uma importante prevalência da autopercepção negativa da saúde de idosos em Minas Gerais. Em equivalência, a pesquisa realizada com dados da PNAD por Lima, Barreto, Giatti (2003, apud BORGES et al., 2014) mostra uma classificação de apenas 24,5\% da população idosa brasileira considerando a sua saúde como "boa" ou "muito boa"; além do estudo de Silva, Pinto Junior e Vilela (2014), que converge com os dados encontrados na presente pesquisa, no estudo de Borges et al. (2014) e no estudo de Confortin et al. (2015). Existe, ainda, uma pesquisa pioneira acerca da autopercepção da saúde em idosos institucionalizados em Natal, que nos faz refletir sobre o grande quantitativo de idosos que observam a sua saúde negativamente em decorrência de fatores de saúde e aspectos variados (JEREZ-ROIG et al., 2016). Com isso, Confortin et al. (2015) afirmam que a categorização da autopercepção da saúde não é homogênea, podendo existir, nas regiões estudadas, variáveis tanto socioeconômicas quanto demográficas que podem interferir na prevalência da autopercepção da saúde.

Os resultados sugerem, para esta amostra, uma percepção do envelhecimento associada à consciência dos benefícios positivos do envelhecimento dentro dos domínios multidimensionais da autopercepção do envelhecimento. Dessa forma, os idosos creem que o envelhecimento proporciona aspectos mais positivos do que negativos, o que pode influenciar na sua capacidade funcional (ROCHA; GOMES; SCHWANKE, 2012). A percepção mais positiva do que negativa pode estar conectada à capacidade de resiliência dos idosos, os quais, apesar das perdas e declínios, tendem a reorganizar-se e maximizar os aspectos positivos (FERREIRA; SANTOS; MAIA, 2012).

A autopercepção da saúde na visão dos idosos deste estudo, em sua maior parte, é considerada boa, embora a maioria apresente condições crônicas de saúde. Silva e Menezes (2007) consideram que a autopercepção da saúde é uma variável bastante consistente devido à sua forte associação com a mortalidade, e a investigação dos fatores determinantes da autopercepção da saúde, junto com algumas mudanças, podem alterar a autopercepção da saúde e a mortalidade nos idosos.

A autopercepção do envelhecimento consiste na autoavaliação dos idosos acerca do processo de envelhecimento e como eles percebem sua vida. Rocha, Gomes e Schwanke (2012) consideram que a autopercepção do envelhecimento é um bom indicador de um envelhecimento bem-sucedido. Ao realizar a associação da autopercepção do envelhecimento e autopercepção da saúde em idosos de um Centro de Convivência do Distrito Federal, verificou-se que a qualidade de vida dos idosos está relacionada à capacidade funcional e à forma como o mais velho percebe a vida e seu meio. São diversos os fatores 
que influenciam na percepção e autoavaliação do idoso com relação à vida, conforme Silva, Pinto Junior e Vilela (2014). A população deste estudo demonstra uma satisfação mais positiva com a vida, apesar dos aspectos negativos vividos, como doenças crônicas e perdas sociais. A autopercepção da saúde e a autopercepção do envelhecimento tornam-se, portanto, indicadores da qualidade de vida que o idoso tem e do seu nível de capacidade funcional. Vale ressaltar, ainda, a afirmativa do estudo de Confortin et al. (2017), que discorre sobre a associação entre as incapacidades e o maior risco de mortalidade. Por isso, torna-se fundamental subsidiar políticas de atenção à saúde do idoso e de estímulo ao envelhecimento ativo.

Apesar de revelar que não houve uma associação estatisticamente significativa, a análise da autopercepção da saúde e da autopercepção do envelhecimento caminham juntas no sentido de buscar a compreensão acerca da qualidade de vida dos idosos. Indagar esses dados amplia o debate sobre as políticas de promoção, prevenção e proteção, de modo a planejar e implementar ações que beneficiem a qualidade de vida da população idosa. O presente estudo permeia a interação entre saúde e envelhecimento positivamente, desmistificando ideologias anteriores acerca da temática.

Os resultados do estudo devem ser interpretados à luz de algumas limitações. O delineamento transversal não indica as relações de causas das variáveis investigadas, mas sinaliza a extensão das associações e permite gerar hipóteses para o desenvolvimento da área em estudo. A utilização de variáveis autorreferidas pode ocasionar viés de informação; entretanto, destaca-se que foram utilizados também instrumentos padronizados.

\section{Considerações finais}

Observou-se não haver relação entre a autopercepção da saúde e a autopercepção do envelhecimento, pois a maioria dos idosos avaliou a autopercepção da saúde como boa e as percepções negativas de saúde não estão relacionadas com a presença de doenças. Assim sendo, as perdas ocasionadas nessa etapa da vida não são, necessariamente, um reflexo da autopercepção da saúde.

Os resultados obtidos detectaram uma autopercepção positiva da saúde entre os idosos, o que demonstra a capacidade de adaptação às limitações decorrentes do processo de envelhecimento, e uma autopercepção do envelhecimento razoável e boa, mostrando que o envelhecimento pode ser um processo que agrega benefícios, como boas experiências e sabedoria. Tornam-se necessários para a construção do saber científico a continuidade dos estudos e o incentivo à 
realização de novas pesquisas em gerontologia, para a obtenção de informações que colaborem com o processo de envelhecimento saudável, proporcionando uma melhor qualidade de vida para os idosos e um aprendizado sobre como beneficiar e cuidar desse grupo populacional em evidente crescimento.

\author{
ASSOCIATION BETWEEN \\ THE SELF-PERCEPTION OF AGING \\ AND THE SELF-PERCEPTION OF HEALTH
}

abstract

The self-perception of health is a multidimensional and subjective, determinant factor for an individual to analyze their own health, considered part of evidence of functional decline and mortality. The objective of this study was to investigate the association between self-perception of aging and self-perception of health. It is a cross-sectional, descriptive and associative study. The sample consisted of 224 individuals over 60 years of age, of both sexes, with 64.4\% female and $35.5 \%$ male individuals, resident in the Federal District. Data collection was performed using a demographic questionnaire and the Aging Self-Perception Questionnaire (QAPE), Brazilian version. Descriptive, comparative analysis group and correlations were performed. It was verified that there was no significant statistical difference between the self-perception of aging and the self-perception of health, however, the data shows that the better the self-perception of health, the better the self-perception of aging.

keywords

Self-Perceived. Health. Aging. Elderly.

referências

BARKER, Maja et al. Cross-sectional validation of the Aging Perceptions Questionnaire: a multidimensional instrument for assessing self-perceptions of aging. BioMed Central Geriatrics, v. 7, n. 9, p. 1-13, Apr. 2007.

BORGES, Aline Morás et al. Autopercepção de saúde em idosos residentes em um município do interior do Rio Grande do Sul. Revista Brasileira de Geriatria e Gerontologia, Rio de Janeiro, v. 17, n. 1, p. 79-86, mar. 2014.

CARDOSO, Maria Cristina et al. Análise da capacidade funcional dos idosos de Porto Alegre e sua associação com autopercepção de saúde. Estudos Interdisciplinares sobre o Envelhecimento, Porto Alegre, v. 17, n. 1, p. 111-124, maio 2012 
CONFORTIN, Susana Cararo et al. Autopercepção positiva de saúde em idosos: estudo populacional no Sul do Brasil. Cadernos de Saúde Pública, Rio de Janeiro, v. 31, n. 5, p. 1049-1060, maio 2015.

Condições de vida e saúde de idosos: resultados do estudo de coorte EpiFloripa Idoso. Revista Epidemiologia e Serviços de Saúde, Brasília, v. 26, n. 2 p. 305-317, jun. 2017.

FECHINE, Basílio Rommel Almeida; TROMPIERI, Nicolino. Processo de envelhecimento: as principais alterações que acontecem com o idoso com o passar dos anos. InterSciencePlace, Campos dos Goytacazes, v. 1, n. 20, p. 106-132, jan./mar. 2012.

FERREIRA, Camomila Lira; SANTOS, Lúcia Maria Oliveira; MAIA, Eulália Maria Chaves Resiliência em idosos atendidos na Rede de Atenção Básica de Saúde em município do nordeste brasileiro. Revista da Escola de Enfermagem da USP, São Paulo, v. 46, n. 2, p. 328-334, abr. 2012

FERREIRA, Olívia Galvão Lucena et al. Envelhecimento ativo e sua relação com a independência funcional. Revista Texto \& Contexto Enfermagem, Florianópolis, v. 21, p. 513-518, jul./set. 2012. Suplemento 3.

FIEDLER, Mariarosa Mendes; PERES, Karen Glazer. Capacidade funcional e fatores associados em idosos do Sul do Brasil: um estudo de base populacional. Cadernos de Saúde Pública, Rio de Janeiro, v. 24, n. 2, p. 409-415, fev. 2008.

FREITAS, Daniela Helena Machado de et al. Autopercepção da saúde e desempenho cognitivo em idosos residentes na comunidade. Revista de Psiquiatria Clínica, São Paulo, v. 37, n. 1, p. 32-35, 2010.

GUERRA, Ana Carolina Lima Cavaletti; CALDAS, Célia Pereira. Dificuldades e recompensas no processo de envelhecimento: a percepção do sujeito idoso. Ciência \& Saúde Coletiva, Rio de Janeiro, v. 15, n. 6, p. 2931-2940, out. 2010.

JEREZ-ROIG, Javier et al. Autopercepção da saúde em idosos institucionalizados Ciência \& Saúde Coletiva, Rio de Janeiro, v. 21, n. 11, p. 3367-3375, nov. 2016.

MEDEIROS, Sarah Magalhães et al. Fatores associados à autopercepção negativa da saúde entre idosos não institucionalizados de Montes Claros, Brasil. Ciência \& Saúde Coletiva, Rio de Janeiro, v. 21, n. 11, p. 3377-3386, nov. 2016.

MIRANDA, Gabriella Morais Duarte; MENDES, Antonio da Cruz Gouveia: SILVA, Ana Lucia Andrade da. O envelhecimento populacional brasileiro: desafios e consequências sociais atuais e futuras. Revista Brasileira de Geriatria e Gerontologia, Rio de Janeiro, v. 19, n. 3, p. 507-519, jun. 2016.

MOREIRA, Ramon Missias et al. Qualidade de vida, saúde e política pública de idosos no Brasil: uma reflexão teórica. Revista Kairós Gerontologia, São Paulo, v. 16, n. 1, p. 27-38, 2013.

NUNES, Ana Paula Nogueira; BARRETO, Sandhi Maria; GONÇALVES, Luana Giatti. Relações sociais e autopercepção da saúde: Projeto Envelhecimento e Saúde. Revista Brasileira de Epidemiologia, Belo Horizonte, v. 15, n. 2, p. 415-428, mar. 2012.

OFSTEDAL, Mary Beth et al. Self-assessed health expectancy among older Asians: a comparison of Sullivan and multistate life table methods. Report n. $03-60$. Ann Arbor: Population Studies Center, 2002. (Comparative Study of the Elderly in Asia Research Reports Series).

RABELO, Dóris Firmino et al. Qualidade de vida, condições e autopercepção da saúde entre idosos hipertensos e não hipertensos. Revista Kairós Gerontologia, São Paulo, v. 13, n. 2, p. 115-130, nov. 2010

RIBEIRO, Liliane da Consolação Campos; ALVES, Pâmela Braga; MEIRA, Elda Patrícia de. Percepção dos idosos sobre as alterações fisiológicas do envelhecimento. Revista Ciência, Cuidado e Saúde, Diamantina, v. 8, n. 2, p. 220-227, abr./jun. 2009. 
ROCHA, Laura Maria Brenner da; GOMES, Irênio; SCHWANKE, Carla Helena Augustin. Tradução e adaptação cultural do APQ-Aging Perceptions Questionnaire para a língua portuguesa brasileira. Revista Brasileira de Geriatria e Gerontologia, Rio de Janeiro, v. 15, n. 2, p. 233-242, 2012

ROCHA, Marianne Pinheiro da; VIEBIG, Renata Furlan; LATTERZA, Andrea Romero. Imagem corporal em idosos: influências dos hábitos alimentares e da prática de atividade física. Lecturas Educación Física y Deportes, Buenos Aires, v. 15, p. 1-1, mar. 2012.

SILVA, Isnanda Tarciara da; PINTO JUNIOR, Elzo Pereira; VILELA, Alba Benemérita Alves. Autopercepção de saúde de idosos que vivem em estado de corresidência. Revista Brasileira de Geriatria e Gerontologia, Rio de Janeiro, v. 17, n. 2, p. 275-287, 2014.

SILVA, Thiago Rodrigo da; MENEZES, Paulo Rossi. Autopercepção de saúde: um estudo com idosos de baixa renda de São Paulo. Revista de Medicina, São Paulo, v. 86, n. 1, p. 28-38, jan./mar. 2007.

SILVEIRA, Erika Aparecida; DALASTRA, Luana; PAGOTTO, Valéria. Polifarmácia, doenças crônicas e marcadores nutricionais em idosos. Revista Brasileira de Epidemiologia, Goiânia, v. 17, n. 4, p. 818-829, out./dez. 2014.

UNITED NATIONS. Department of Economic and Social Affairs/Population Division. World Population Prospects: the 2008 revision. New York: United Nations, 2009.

Data de Submissão: 21/06/2016

Data de Aprovação: 17/09/2018 